\title{
Addictions with Co-occurring Problems: Statistics and Challenges
}

\author{
Marie-Josée Fleury ${ }^{1^{*}}$ and Serge Brochu ${ }^{2^{* *}}$ \\ ${ }^{1}$ Montreal Addiction Rehabilitation Centre - University Institute, McGill University, Canada \\ ${ }^{2}$ Montreal Addiction Rehabilitation Centre - University Institute, Université de Montréal, Canada \\ "Corresponding author: Marie-Josée Fleury Montreal Addiction Rehabilitation Centre - University Institute, Canada, Tel: 514-385-3490 ext.: 3112; Fax: 514-385-4685; \\ Email: flemar@douglas.mcgill.ca \\ ${ }^{* *}$ Corresponding author: Dr. Serge Brochu, Montreal Addiction Rehabilitation Centre - University Institute, Université de Montréal, Canada, Tel: 514-343-7469; Fax: \\ 514-343-2098; Email: serge.brochu@umontreal.ca
}

Received date: June 19, 2014; Accepted date: June 26, 2014; Published date: June 30, 2014

Copyright: (C) 2014 Fleury MJ and Brochu S. This is an open-access article distributed under the terms of the Creative Commons Attribution License, which permits unrestricted use, distribution, and reproduction in any medium, provided the original author and source are credited.

\section{Introduction}

Addiction remains a serious public health concern because of its associated health and related socio-economic impacts. The World Health Organization (WHO) lists interventions to reduce harm from substance abuse as a top priority. In 2009, tobacco and alcohol figured among the 10 leading risk factors internationally in terms of avoidable diseases, and contributed 3.7 and 4.4 percent respectively to the world burden of illness, while illicit substances accounted for 0.9 percent [1]. More than 80 chronic and infectious diseases and injuries - including trauma, suicide, road accidents, neglect and family violence - are associated with the consumption of psychoactive addiction [2]. Pathological gambling also leads to a number of significant problems such as financial hardship, illegal activities and loss of social support [3]. Meanwhile, the global social costs (death, illness and economic burden) of substance abuse have reached alarming levels. Canada estimated such costs - excluding gambling - at \$39.8 billion in 2002 . Productivity losses, premature deaths and illnesses represent nearly two thirds of the social costs, followed by health care (22\%) and legal expenditures (14\%) [4].

People usually experience the onset of mental disorders and addiction at a young age, before they reach 25 in most cases [5]. For the majority of them, addiction becomes a long-term or chronic condition as they experience cycles of recovery, relapse, and repeated treatments. As opposed to other consumers, chronic users generally consume a wider variety of substances, in larger quantities and more frequently $[3,6]$. Among other factors that may lead to chronic addiction are early onset of consumption, delay in seeking treatment, unstable living conditions, and the presence of other health problems such as mental disorders.

Research shows that the co-occurrence of addiction and mental disorders is the rule rather than the exception [7,8]. An epidemiological studies review has shown that from seven to forty-five percent of individuals with alcohol dependence, and from seventeen to fifty-five percent of those with drug dependence, also suffer from a mood or anxiety disorder [9]. Another report concluded that the most common psychiatric diagnosis among substance users was personality disorder (50-90\%), followed by affective disorders (20-60\%) and psychotic disorders (20\%). Between ten and fifty percent of patients exhibited more than one psychiatric or personality co-morbidity disorder [10]. Individuals suffering from mental disorders smoke at nearly twice the rate of the general population (i.e., $41 \%$ versus $23 \%$ ) with even higher rates among the severely mentally ill and those with additional addictions [11,12]. Eating disorders (particularly bulimia nervosa and binge-eating disorder) have also been associated with comorbid substance abuse [13]. Studies have shown that between twenty and forty percent of women suffering from bulimia reported a history of problems with alcohol, drugs or both $[14,15]$. Recent papers have also identified a link between problem gambling and increased rates of substance use and mental health problems [16,17]. Pathological gamblers are two to three times more likely to experience mental disorders than non-gamblers [18]. The prevalence of co-occurring pathological or problem gambling and alcohol addiction is around twenty-eight percent, while concurrent drug and gambling addictions are present in seventeen percent of cases [19]. Overall, individuals presenting co-occurring disorders have comprehensive needs that require complex, long-term treatment, including a full array of services (e.g., rehabilitation, detox, supported housing) [20].

On the other hand, treatment of people with substance abuse and co-occurring disorders can be a difficult proposition. This group often shows no desire to receive treatment, and motivational therapy and harm reduction initiatives must therefore become essential elements of a rehabilitation plan. In 2002, for example, the ratio of Canadians seeking the advice of a family physician, psychiatrist or psychologist within a 12 -month period were respectively $6.2,1.8$ and 2.3 percent among individuals with substance dependence, 31.6, 14.1 and 10.0 percent among those with mental disorders, and 34.6, 16.1 and 11.0 percent among those with co-occurring disorders [21]. These groups and especially persons with only a substance abuse problem therefore use services well below the optimal level that might address their actual needs $[22,23]$. Individuals with co-occurring disorders are more likely to require the services of hospital emergency departments $[24,25]$, and to have trouble adhering to treatment and medication. They also suffer higher rates of relapse and hospitalization and worse outcomes (in terms, for example, of functional status, employment and quality of life) than people showing only a substance abuse or mental disorder $[6,26]$.

A number of developed countries such as the US, the UK, Canada and Australia have invested significant resources to establish best practices and guiding principles to assist practitioners and policymakers and provide optimal treatment to people with substance abuse and co-occurring disorders $[8,27,28]$. Experts agree on the need to revamp addiction services, which are usually set up to handle addiction primarily as an acute or transitory problem and to treat it as a self-contained illness. Services must therefore become more effective in dealing with chronic and co-occurring addictions [29]. They might need, for example, to re-examine the duration of treatment and definition of successful rehabilitation (based on several years), and provide longer follow-up periods. Research has shown that providing treatment in different settings (i.e., separate facilities to deal with mental health and addiction) results in fragmented and ultimately ineffective care. Individuals receive disparate messages about 
treatment and face discontinuity of care, difficulties in navigating the separate systems, and lack of access to services because of other illnesses. Such conditions seem to suggest that integration of substance-abuse and mental health services has become a necessity.

The general heading of co-occurring disorders comprises a heterogeneous clientele with distinctive profiles - including gender differences - and varied and complex needs requiring high-quality, and usually comprehensive and integrated care to ensure better outcomes. Adapting treatment facilities, implementing coordination mechanisms and tailoring services specifically to address co-occurring disorders would result in more effective treatment. Controlled studies suggest that greater integration of substance-abuse and mental health services lead to more productive interventions. Vertical integration as proposed by the Drake and Mueser model of integrated treatment [30] particularly seems to maximize advantages for the more severe and persistent cases of individuals with co-occurring disorders [31]. There are strong indications that this model is more efficient in addressing such cases than traditional non-integrated services, but further study is needed to validate results. For individuals whose co-occurring disorders are less severe and persistent, the solution could involve step-care approaches [32] and service networks [33], including improved continuum of services by developing coordination mechanisms to prevent fragmentation (e.g., systematic screening, onestop services, collaborative clinical plans, electronic clinical records, case managers, boundary spanners, treatment protocols, shared-care, joint programs, and referral arrangements between organizations). We need to learn more about best practices in the treatment of cooccurring disorders [30] and to commit substantial efforts to successfully integrate services and coordinate care, which implies more flexible, multilevel and variable levels of treatment.

In this special issue, we seek to gain deeper knowledge of addiction with co-occurring problems, as we look at risk factors and adverse outcomes and avenues for practitioners and policy-makers to provide services better suited to the needs of individuals with co-occurring disorders. The issue covers epidemiological, service-use and outcome research, along with one study on treatments and issues related to cooccurring disorders. Reports on co-occurring addictions (i.e. tobacco, alcohol, and drugs, but also problem gambling) are included. Most of the articles in this issue consider mental disorders (e.g. depression, eating disorders, and severe mental disorders) as they relate to addiction in different environments (Australia, Canada and Switzerland). We believe readers of JART will find this issue informative as it sheds new light on the interrelationship between addiction and mental health.

\section{References}

1. World Health Organization (2009) Global health risks. Mortality and burden of disease attributable to selected major risks. Geneva, Switzerland.

2. Rehm J, Taylor B, Room R (2006) Global burden of disease from alcohol, illicit drugs and tobacco. Drug Alcohol Rev 25: 503-513.

3. Williams RJ, Stevens RMG (2011) The social and economic impacts of gambling. Final report prepared for the Canadian Consortium for Gambling Research. Canadian Consortium for Gambling Research: Ontario, Canada.

4. Rehm J, Gnam W, Popova S, Baliunas D, Brochu S, et al. (2007) The costs of alcohol, illegal drugs, and tobacco in Canada, 2002. J Stud Alcohol Drugs 68: 886-895.
5. Le Strat Y, Grant BF, Ramoz N, Gorwood P (2010) A new definition of early age at onset in alcohol dependence. Drug Alcohol Depend 108: 43-48.

6. Dennis ML, Scott CK, Funk R, Foss MA (2005) The duration and correlates of addiction and treatment careers. J Subst Abuse Treat 28 Suppl 1: S51-62.

7. Brunette MF, Mueser KT, Drake RE (2004) A review of research on residential programs for people with severe mental illness and cooccurring substance use disorders. Drug Alcohol Rev 23: 471-481.

8. Rush B, Nadeau L, McMain S, Ogborne A, Goering P, et al. (2002) Best practices - Concurrent mental health and substance use disorders. Health Canada.

9. Jané-Llopis E, Matytsina I (2006) Mental health and alcohol, drugs and tobacco: a review of the comorbidity between mental disorders and the use of alcohol, tobacco and illicit drugs. Drug Alcohol Rev 25: 515-536.

10. European Monitoring Centre for Drugs and Drug Addiction, Annual report 2004: the state of the drugs problem in the European Union and Norway. Co-morbidity. 2000: Luxembourg.

11. Lasser K, Boyd JW, Woolhandler S, Himmelstein DU, McCormick D, et al. (2000) Smoking and mental illness: A population-based prevalence study. JAMA 284: 2606-2610.

12. Rohde P, Lewinsohn PM, Brown RA, Gau JM, Kahler CW (2003) Psychiatric disorders, familial factors and cigarette smoking: I. Associations with smoking initiation. Nicotine Tob Res 5: 85-98.

13. Conason AH, Brunstein Klomek A, Sher L (2006) Recognizing alcohol and drug abuse in patients with eating disorders. QJM 99: 335-339.

14. Beary MD, Lacey JH, Merry J (1986) Alcoholism and eating disorders in women of fertile age. Br J Addict 81: 685-689.

15. Hall RC, Beresford TP, Wooley B, Tice L, Hall AK (1989) Covert drug abuse in patients with eating disorders. Psychiatr Med 7: 247-255.

16. Hodgins DC, Cassidy E (2005) The association between comorbidity and outcome in pathological gambling: a prospective follow-up of recent quitters. J Gambl Stud 21: 255-271.

17. Rush BR, Bassani DG, Urbanoski KA, Castel S (2008) Influence of cooccurring mental and substance use disorders on the prevalence of problem gambling in Canada. Addiction 103: 1847-1856.

18. Alberta Health Services (2009) Problem gambling, mental health and suicide. A literature review, A.a.M. Health, Edmonton, Alberta, Canada.

19. Lorains FK, Cowlishaw S, Thomas SA (2011) Prevalence of comorbid disorders in problem and pathological gambling: systematic review and meta-analysis of population surveys. Addiction 106: 490-498.

20. Treatment of persons with severe mental illness and substance use disorders in addiction programs. The Drug and Alcohol Forum.

21. Urbanoski KA, Rush BR, Wild TC, Bassani DG, Castel S (2007) Use of mental health care services by Canadians with co-occurring substance dependence and mental disorders. Psychiatr Serv 58: 962-969.

22. Rush BR, Bassani DG, Castel S, Wild TC (2010) The epidemiology of cooccurring substance use and other mental disorders in Canada: prevalence, service use, and unmet needs. Toronto: University of Toronto Press.

23. Compton MT, Weiss PS, West JC, Kaslow NJ (2005) The associations between substance use disorders, schizophrenia-spectrum disorders, and Axis IV psychosocial problems. Soc Psychiatry Psychiatr Epidemiol 40: 939-946.

24. Curran GM, Sullivan G, Williams K, Han X, Allee E, et al. (2008) The association of psychiatric comorbidity and use of the emergency department among persons with substance use disorders: an observational cohort study. BMC Emerg Med 8: 17.

25. Curran GM, Sullivan G, Williams K, Han X, Collins K, et al. (2003) Emergency department use of persons with comorbid psychiatric and substance abuse disorders. Ann Emerg Med 41: 659-667.

26. Dennis M, Scott CK (2007) Managing addiction as a chronic condition. Addict Sci Clin Pract 4: 45-55. 
Citation: Fleury MJ, Brochu S (2014) Addictions with Co-occurring Problems: Statistics and Challenges. J Addict Res Ther S10: S10-e001. doi: 10.4172/2155-6105.S10-e001

Page 3 of 3

27. United Kingdom Department of Health (2002) Mental Health Policy. Implementation Guide. Dual Diagnosis Good Practice Guide. United Kingdom Department of Health: London.

28. National Drug and Alcohol Research Centre (2001) National Comorbidity Project. National Drug Strategy and National Mental Health Strategy: Commonwealth of Australia.

29. Kelly JF, William L (2011) Addiction recovery management. Theory, research and practice. US: Humana Press.

30. Drake RE, Mueser KT, Brunette MF (2007) Management of persons with co-occurring severe mental illness and substance use disorder: program implications. World Psychiatry 6: 131-136.
31. Donald M, Kavanagh D (2005) Integrated versus non-integrated management and care for clients with co-occurring mental health and substance use disorders: a qualitative systematic review of randomised controlled trials. Soc Sci Med 60: 1371-1383.

32. Sobell MB Stepped care for alcohol problems: An efficient method for planning and delivering clinical services. In Tucker JA, Donovan DM, Marlatt, G.A. Changing addictive behavior: Bridging clinical and public health strategies. Guilford PressNew York, NY, US: Guilford Press.

33. Fleury MJ (2006) Integrated service networks: the Quebec case. Health Serv Manage Res 19: 153-165. 